\title{
Optimal Operation of Energy Hub with high penetration of renewable energy sources
}

\author{
Patrick Sunday Onen ${ }^{1}$, Geev Mokryani ${ }^{2}$, and Rana H.A. Zubo ${ }^{3}$ \\ \{p.s.onen@bradford.ac.uk,g.mokryani@bradford.ac.uk, r.h.a.zubo@ntu.edu.iq \} \\ University of Bradford, $\mathrm{Uk}^{1,2}$,Northern Technical University, $\mathrm{Iraq}^{3}$
}

\begin{abstract}
Integration of electricity and gas networks increases system flexibility while also increasing energy efficiency. In such a connected infrastructure, the energy hub (EH) plays a critical role in energy production, conversion, and storage. This research provides a new method to EH operation that takes into account renewable energy sources (RESs) and battery energy storage systems (BESS). The suggested model's goal is to reduce total energy costs by optimising total energy prices based on hourly power and heat demand while taking into account hub restrictions. The impact of energy prices, load demands, RESs, and BESS on the EH model's performance has also been explored. Three operational case studies based on distinct EH structures were used to investigate the optimization problem. The CPLEX solver in the general algebraic modelling system (GAMS) software is used to solve a Mixed integer linear programming (MILP) formulation for the optimization problem. The simulation findings demonstrate a decrease in the cost of market-purchased energy.
\end{abstract}

Keywords: Energy hub, optimal operation, Renewable energy source, Battery energy storage system, Energy costs.

\section{Introduction}

\section{A. Background and Motivation}

Growing worldwide energy consumption and the environmental implications of fossil fuels in traditionally operated power plants have heightened the clamour for a shift to more environmentally benign and economically viable renewable energy sources. [1,2]. Furthermore, electricity is not the only energy consumed by consumers for their daily activities, heating is also required by the consumers too. However, the electricity, gas, and heating energy infrastructure have traditionally operated separately which has led to lower efficiency of the system and a higher operational cost with greater emission to the environment. Hence, Multivector energy system integration can solve the issue by combining different sources of energy together in order to complement each other to enhance the system reliability, flexibility, sustainability and reduce energy costs $[3,4]$. 
In addition, when compared to a typical power plant, the integration of combined heat and power (CHP) with the electricity network has grown tremendously to meet the consumer's electrical and heat demands concurrently from a single gas source with minimal emissions and energy expenditures. The EH idea, in which numerous energy carriers are converted, conditioned, and stored to meet the end user's demand, can be used to efficiently implement the coupling of different energy infrastructure. As a result, the economy of energy systems will be boosted by enhancing energy efficiency, lowering fuel consumption, and improving system flexibility, all of which are essential factors in the integration of renewable energy sources. Furthermore, the high shares of RESs generation into the EH systems, will reduce the amount of energy that will be purchased from the market. Hence, reduced the total energy costs.

\section{Literature review}

EH is a relatively new concept in energy system integration, and a promising approach to implementing future energy system integration. Recent studies on the optimal operation of EH have taken a variety of approaches, each with its own set of goals and constraints. During a 'Future energy network vision' project at ETH Zurich in 2007, Ref [5] was the first to explain the meaning and the EH idea. The authors of $[6,7]$ look at the challenge of determining the best power flow for multi-energy carriers using a variety of renewable energy sources. Ref [5] investigated a general model for power flow as well as an optimization technique for EHs with multiple energy carriers, such as district heating, natural gas, and electrical networks. [8] looked at deterministic and stochastic situations, as well as the best way to operate an EH with a wind turbine (WT), energy storage, and demand response. The operation of EH in the face of responsive loads and stochastic wind generation was investigated in ref [9]. [10] proposed an economic dispatch model of eleven interconnected $\mathrm{EH}$ to investigate the impact of wind energy uncertainty. Past studies on the EH operations have been implemented based on different consumer's need such as residential [11], commercial [16], industrial [19], and agricultural consumers [6]. From the above analysis, this paper mainly solves the optimal operation problem of the EH model in relation to energy costs, considering different hourly electricity prices and load demand. Precisely, the proposed EH model can provide electricity and heat loads simultaneously using RESs generation from WT and PV, CHP, GB, and energy purchased from the market. The model can connect and interchange multiple types of energy to get the best energy flow with the lowest running expenses. Finally, the model in this research employs BESS to improve system efficiency. BESS is a critical component of increasing power supply dependability and flexibility. Indeed, the BESS has been extensively employed in operations and planning to minimise investment costs and the cost of acquiring power from the system, as well as to accommodate significant shares of renewable energy output at peak production capacity, all with the purpose of enhancing economic efficiency.

\section{B. Aim and objectives}

This work aims to "perform and analyze optimal operations of EH with high penetration of renewable energy resources.

This work is based on the following objectives:

- Mathematically modelling of optimal operation of EH to optimize total energy costs based on hourly demand of electricity and heat using the MILP model to mitigate the computational burden in the optimal operation problem.

- Examine the impact of load demand and energy prices on the EH model's performance.

- Investigate the impact of high penetration of RESs and BESS on the performance of the EH model. 


\section{Paper organization}

The approach and problem solving in this paper are organized in the following manner. Section 2 represents the basic concept of the EH, the EH model and the description of the proposed EH structure. Section 3 delves into the mathematical model, including objective functions (OFs) and mathematical limitations such as the EH model's input and output power balance, equipment conversion limits, and system power capacity limits. Section 4 depicts the optimal operation problem answered in simulation results and comments using the GAMS programming languages. Section 5 concludes with findings and future research directions.

\section{Basic concept of EH}

The EH idea is a compelling representation of how numerous energy carriers are converted, conditioned, and stored to improve the system's energy efficiency and resource use. In a multi vector energy system, an EH is an input-output dual-port model that describes energy supply, load demand, network switching, and coupling interactions [11]. The coupling matrix is widely used in multi vector energy system planning and operation analysis to synergies various energy carriers, non-hierarchical structure, and integrated management of different energy infrastructures that have traditionally operated independently in the past, and it is widely used in multi vector energy system planning and operation analysis to synergies various energy carriers, non-hierarchical structure, and integrated management of different energy infrastructures that have traditionally operated independently in the past. The EH coupling matrix, which describes the input energy and output load port, can be used to represent various coupling relationships such as conversion, storage, and transmission between various forms of energy such as electricity, heat, and gas, and can be useful in the planning and operation of EH [12].

\section{- Energy Hub Model}

A generic model of EH applies a coupling matrix $\mathrm{C}$, which consist of various coupling factors as shown in Fig. 1 below [13, 14]

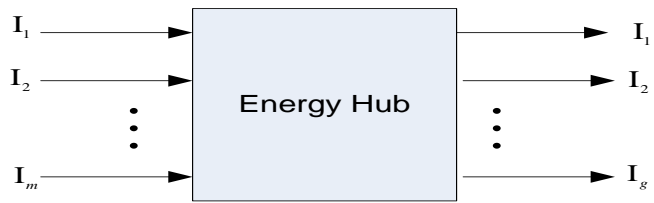

Fig.1 Energy carriers Transformation through an EH [14].

The coupling matrix can be expressed in Eq. (1):

$$
\left[\begin{array}{c}
I_{1} \\
I_{2} \\
\vdots \\
I_{m}
\end{array}\right]\left[\begin{array}{cccc}
C_{11} & C_{12} & \cdots & C_{1 n} \\
C_{21} & C_{22} & \cdots & C_{2 n} \\
\vdots & \vdots & \ddots & \vdots \\
C_{m 1} & C_{m 2} & \cdots & C_{m n}
\end{array}\right]=\left[\begin{array}{c}
L_{1} \\
L_{2} \\
\vdots \\
L_{n}
\end{array}\right]
$$

where $\mathrm{C}_{\mathrm{mn}}$ denotes the coupling coefficients between input carrier I and output carrier $\mathrm{L}$, and $\mathrm{m}$ and $\mathrm{n}$ denote the input and output carrier numbers, respectively [15].

\section{- Proposed Energy Hub}

To begin, the proposed EH model is based on energy and heating load demand. At the input ports connected to the energy infrastructures, various sources of energy are received, and the energy is delivered at the output ports. Converter technologies such as TR, CHP, GB, and BESS are used to convert and condition various sources of energy. The energy vectors at the input 
ports of the EH are purchased from the market and renewable energies (WT and PV). The diagram in figure 2 illustrate the proposed EH to enhance operational flexibility and achieve effectiveness of energy supply. The input forms of energy are converted through the converter technologies to supply the load demands. The electrical load demand is supplied electricity via TR, RESs and the CHP. While the CHP and GB supplies the heating load demand. Lastly, the BESS is used in this model to improve efficiency.

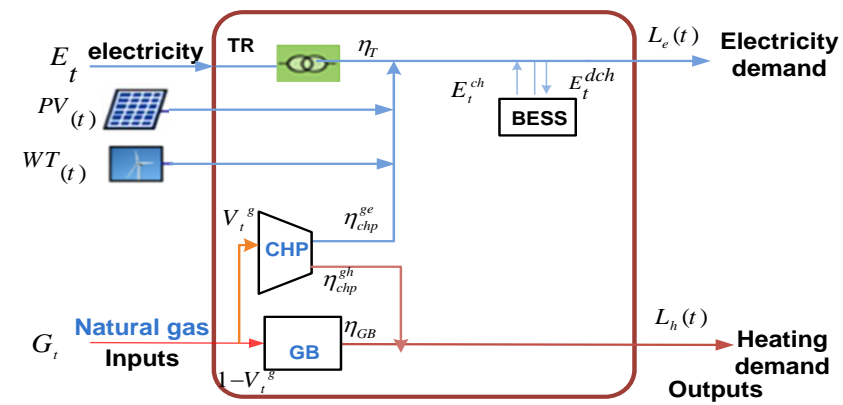

Fig.2 Proposed Energy hub structure.

\section{Mathematical Model}

\section{A. Object function}

The problem's purpose is to reduce EH's total energy costs. As a result, Eq. (2) is the minimum objective function of the cost of buying energy from the market each day, with Et and Gt indicating the amount of electricity and natural gas purchased from their respective networks at each hour of the day.

Min OF $\sum_{t=1}^{N h} \lambda_{t}^{e} E_{t}+\lambda_{t}^{g} G_{t}$

where $\lambda_{t}^{e}$ is the price of electricity per hour t and $\lambda_{t}^{g}$ is the constant natural gas price per hour t; and the $\mathrm{Nh}$ is the total number of hours a day $(24 \mathrm{~h})$.

\section{B. The constraints}

The mathematical model should include the following restrictions to ensure the smooth functioning and power of the equipment in the proposed $\mathrm{EH}$ model.

\section{- Energy balance constraints of the EH}

Based on the operating principles of the $\mathrm{EH}$, the relationship between the energy demands $\mathrm{L}_{\mathrm{e}}$ $(\mathrm{t}), \mathrm{L}_{\mathrm{h}}(\mathrm{t})$ and energy purchased from the network $E_{t}$ and $G_{t}$ at time $\mathrm{t}$ is expressed in (3) \& (4):

$$
\begin{aligned}
& \eta_{e e}^{T} E_{t}+\eta_{g}^{C h p} G_{t}+P w(t)+P V(t)+E_{t}^{d c h}-E_{t}^{c h}=L_{e}(t) \\
& \eta_{g h}^{C h p} G_{t}+\eta_{g h}^{G B} G_{t}=L_{h}(t)
\end{aligned}
$$

where $E_{t}^{c h}$ and $E_{t}^{d c h}$ are the charge and discharge power of the BESS at hour t, respectively; $\mathrm{PV}(\mathrm{t})$ and $\mathrm{PW}(\mathrm{t})$ are the electrical power produced from RESs at hour $\mathrm{t}$. The $\mathrm{L}_{\mathrm{e}}(\mathrm{t})$ and $\mathrm{L}_{\mathrm{h}}(\mathrm{t})$ are the output load demand of electricity and heat. The $\eta_{g_{e}}^{C h p} G_{t}$ and $\eta_{g_{h}}^{C h p} G_{t}$ are the gas to electricity and heat conversion efficiency of the CHP. While the $\eta_{g h}^{G B}$ is the conversion efficiency of the GB and the $\eta_{e e}^{T}$ is the efficiency of the TR.

\section{- Battery Storage system (BESS) constraint}


Within a 24-hour cycle, the features of the electricity price and renewable energy generation change. During peak hours, the price of electricity is higher, and vice versa. As a result, as demonstrated in Eq. 1, the total energy stored and sent back to the EH system must be balanced (5). Eqs. (6) and (7) took into account the BESS limits, which are the battery's lowest to maximum charging and discharging power capacities, respectively (i.e., the entire storage capacity of BESS must be smaller than the maximum capacity of BESS) [16]. Depending on the model operation, the BESS can work in charge or discharge mode and can be charged from the system. To prevent charge and discharge at the same time, the binary variables of charge and discharge are used in (8).

$$
\begin{aligned}
& \sum_{t=1}^{N h} \eta_{B E S S} \cdot I_{B E S S}^{c h}(\mathrm{t}) \cdot E_{B E S S}^{c h}(\mathrm{t})=\sum_{t=1}^{N h} I_{B E S S}^{d c h}(\mathrm{t}) \cdot E_{B E S S}^{d c h}(\mathrm{t}) \\
& E_{\min }^{c h} I_{t}^{c h} \leq E_{t}^{c h} \leq E_{\max }^{c h} I_{t}^{c h} \\
& E_{\min }^{d c h} I_{t}^{d c h} \leq E_{t}^{d c h} \leq E_{\max }^{d c h} I_{t}^{d c h} \quad \text { (7) } I_{t}^{c h}+I_{t}^{d c h} \leq 1 \quad I_{t}^{c h}, I_{t}^{c h} \in\{0,1\}
\end{aligned}
$$

Similarly, the BESS must be less than the max. capacity of BESS, $E_{B E S S}^{\max }$, with constraints as expressed in (9) and (10) below:

$$
\begin{gathered}
0 \leq E_{B E S S}(t) \leq E_{B E S S}^{\max } \\
E_{B E S S}(t)=E_{B E S S(t-1)}^{c h}+E_{B E S S}^{c h}(t)-E_{B E S S}^{d c h}(t) \\
\forall t \in N h
\end{gathered}
$$

\section{- The constraints of power flow}

The optimal operation of the EH model is to control the power flow of the TR, CHP, BESS, and GB devices. Therefore, the conversion limits of the mentioned devices at time $t$ are expressed below:

$$
\begin{aligned}
& 0 \leq \eta C H P(t) \leq 1 \\
& 0 \leq \eta T R(t) \leq 1 \\
& 0 \leq \eta G B(t) \leq 1
\end{aligned}
$$

\section{- The constraints of power purchased from the market.}

The capacity and energy stored in the system limit the system's supply capacity. Consequently, the constraint utilized are represented in Eq. (14) and (15):

$$
\begin{aligned}
& E(t) \leq E^{\max } \\
& G(t) \leq G^{\max }
\end{aligned}
$$

where $E^{\max }$ and $G^{\max }$ are the max. allowable electrical energy and natural gas, respectively. The purchased energy within a day to the proposed EH is limited by the energy that can be supplied from the electricity and gas network mentioned above are expressed in Eq. (16) and (17) respectively.

$$
\begin{aligned}
& \sum_{t=1}^{N h} E(t) \leq E_{\max }^{e} \\
& \sum_{t=1}^{N h} G(t) \leq E_{\max }^{g}
\end{aligned}
$$

where $E_{\max }^{e}$ and $E_{\max }^{g}$ are the supplied power from the electricity and natural gas network.

Wind speed constraints

The O/P of WT a day is.

$$
P_{\text {wind }}^{e}(w(t))=\left\{\begin{array}{l}
P_{\text {out }}^{w} \\
\frac{\omega(t)-\omega_{i}^{\text {cut }}}{\omega_{r}^{w}-\omega_{i}^{\text {cut }}}\left(P_{\text {out }}^{w}\right) \omega_{i}^{\text {cut }} \leq \omega(t) \leq \omega_{r}^{w} \\
0
\end{array}\right.
$$


$\omega_{r}^{w} \leq \omega(t) \leq \omega_{o}^{\text {cut }}, \omega(t) \leq \omega_{i}^{\text {cut }}$ or $\omega(t) \geq \omega_{o}^{\text {cut }}$

Eq. 1 shows the limits imposed by this dependency (18).

The output power of the WT is computed at time $t$ using the equation. When considering varied levels of wind speed, this output power can occur in a variety of ranges. $\omega_{i}^{\text {cut }}, \omega_{o}^{\text {cut }}, \omega_{r}^{W}, \omega(\mathrm{t})$ and $\left(P_{\text {out }}^{W}\right)$ represent cut in and cut out speed state of WT, rate of speed of WT, hourly wind speed at time $t$ and power output, respectively

PV constraint

The PV panels' output power is determined by the sun irradiation at different times of the day. Eq. (19) shows the output power constraints from the PV panels.

$P_{P V}^{e}\left(S(t)=P V_{\text {array }} d f^{P V}\left(\frac{S i(t)}{S i, S T C}\right)\left[1+\alpha \rho\left(T_{c}-T_{c S T C}\right)\right]\right.$

The $\mathrm{PV}_{\text {array }}(\mathrm{kW})$ represent the $\mathrm{PV}$ rated capacity of array. Where $d f^{P V}, \alpha \rho, S i, S T C, T c, S T C, T c$ represent derating factor, temperature coefficient, solar irradiance and temperature of PV cell at standard test condition, and temperature of PV cell respectively. WT and PV panels create power based on random variables such as wind speed and solar irradiation.

\section{Reults and discussion}

The optimization of input power and energy expenses of the EH for each day is computed to highlight the advantages of the proposed $\mathrm{EH}$ with the mathematical model described in section 3. Additionally, the effect and role of high penetration of RES and BESS on the operation of the $\mathrm{EH}$ are also compared in three model cases, as presented in Table 1.

Table 1. Calculated Cased study of the EH structure

\begin{tabular}{|c|c|c|c|c|c|}
\hline Case & Input & Converter & $\begin{array}{l}\text { BES } \\
\text { S }\end{array}$ & RES & $\begin{array}{l}\text { Outp } \\
\text { ut }\end{array}$ \\
\hline 1 & $\mathrm{E}, \mathrm{G}$ & $\begin{array}{l}\text { CHP, TR, } \\
\text { GB }\end{array}$ & - & - & E, $\mathrm{H}$ \\
\hline 2 & E, G & $\begin{array}{l}\text { CHP, TR, } \\
\text { GB }\end{array}$ & - & $\begin{array}{l}\text { PW, } \\
\text { PV }\end{array}$ & $\mathrm{E}, \mathrm{H}$ \\
\hline 3 & $\mathrm{E}, \mathrm{G}$ & $\begin{array}{ll}\text { CHP, } & \text { TR, } \\
\text { GB }\end{array}$ & $\begin{array}{l}\text { BES } \\
\text { S }\end{array}$ & $\begin{array}{l}\text { PW, } \\
\text { PV }\end{array}$ & E, $\mathrm{H}$ \\
\hline
\end{tabular}

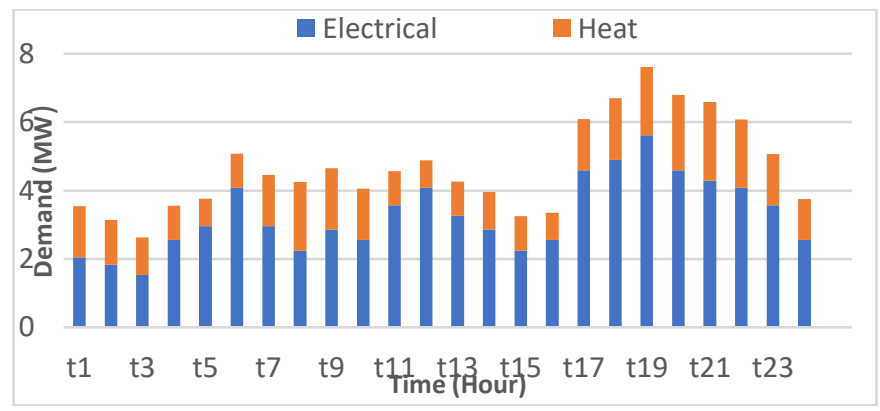

Fig.3 The hourly electrical and heating output load of the EH

Assumptions and database analysis

Electrical and heating demand

During day-to-day operations, the planned EH's energy load need fluctuates dramatically. As a result, in this research, the typical daily electrical and heating load characteristics are depicted 
in Fig (3). Where the greatest load output power is at 7 p.m. with a $5.5 \mathrm{MW}$ capacity and the minimum load power is at 3 a.m. with a $1.5 \mathrm{MW}$ capacity, equal to a 26 percent percentage while achieving the maximum. With a power capacity of $2.38 \mathrm{MW}$, the heating load is rather small.

Gas and Electrical energy prices

Currently, gas prices have fluctuated slightly in the near term, so it is roughly $5.5 \$ / \mathrm{KWh}$ on a regular day. Electricity rates fluctuate a lot over time and are determined by the market. Figure 1 depicts the highest electricity price per hour in this article. In figure (4), electricity price reaches its highest price at 7PM which is the higher assumed peak time in this paper. It is assumed that at 7PM most of consumers are back home from their daily activities and the demand of electricity has gone up due to many customers using different appliances at the same time. Therefore, it is logical to state that when the demand is high the price will also be high as it can be seen from both figure (3) and (4) respectively. Furthermore, it can be seen from both figure (3) and (4), that at 3AM in the early hours of the day, when most of the consumers, are assumed to be sleeping, the loads demand is at its lowest state, here the price of electricity at that period is also at its lowest price. Demand response could be incorporated in future studies to help shift consumers load demand from 7PM (peak time and highest electricity price) to $3 \mathrm{AM}$ (lowest demand time and less electricity price).

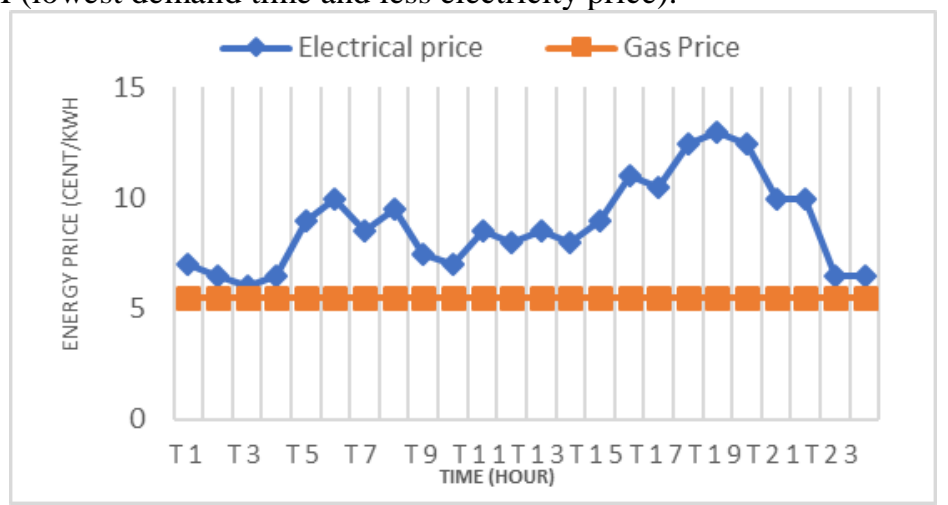

Fig.4 Electricity and gas price

Table 2 shows that energy conversion equipment has a high efficiency. CHP has a total conversion efficiency of 0.85 , with $40 \%$ of the energy converted to electricity and $50 \%$ converted to heat. The efficiency of the GB is 0.9 and the efficiency of the BESS is 0.9. The TR has the highest efficiency of $95 \%$.

Table 2. Efficiency data of equipment

\begin{tabular}{|l|l|l|l|l|}
\hline$\eta_{T}$ & $\eta_{c H P}^{g e}$ & $\eta_{C H P}^{g_{h}}$ & $\eta_{G B}$ & $\eta_{\text {BESS }}$ \\
\hline 0.95 & 0.4 & 0.45 & 0.9 & 0.9 \\
\hline
\end{tabular}

Table 3 shows the equipment's power restrictions, as well as the capacity of the BESS and the system, to ensure the equipment's correct operation and ability to supply the system. Finally, take into account the previously stated assumptions. The CPLEX solver of GAMS software was used to solve the proposed MILP problem in this paper, which is one of the most powerful solvers of MILP problems. The proposed optimization problem takes around 62 seconds to solve.

Table 3 Capacity data of equipment and the system

\begin{tabular}{|l|l|l|l|}
\hline Parameters & Value & Parameters & Value \\
\hline $\begin{array}{l}E_{\max }^{e} \\
(\mathrm{MWh})\end{array}$ & 100 & $G^{\max }(\mathrm{MW})$ & 5 \\
\hline $\begin{array}{l}E_{\max }^{g} \\
(\mathrm{MWh})\end{array}$ & 50 & $E_{B E S S}^{\max }(\mathrm{MW})$ & 0.15 \\
\hline
\end{tabular}




\begin{tabular}{|l|l|l|l|}
\hline$E^{\max }(\mathrm{MW})$ & 10 & $E_{\min }^{\text {ch/dch }}$ & 0 \\
\hline
\end{tabular}

\section{Results Analysis and Discussion}

The model computed with the assumed hypothetical parameters. The results determine the energy flow in the devices in all the operations throughout the hours of the day. The minimum load of energy flow is during 3AM in the early hours of the day (the electricity load is at $1.5 \mathrm{MW}$, the heat load is at $1.13 \mathrm{MW}$ ), and the maximum load is at $7 \mathrm{PM}$ which is the $19^{\text {th }}$ hour of the day (the electricity load reaches $5.5 \mathrm{MW}$, the heat load is at $2.38 \mathrm{MW}$ ) as shown in Figure 3.

Case 1: At peak load times (between 5 a.m., 7 a.m., 9 a.m., and 5 p.m., 10 p.m.), CHP was chosen to offer additional load with a high capacity of 4.0 MW, because the electricity price was 13 cents per $\mathrm{kWh}$ and the gas price was just 5.5 cents per $\mathrm{kWh}$. Hence, it is cheaper to buy natural gas which enable the CHP to simultaneously meet the EH electrical and heat demand. 1.0 MW was obtained by the transmission capacity through. Although, the CHP even at its maximum capacity was not able to meet the assumed high peak load demand of $5.5 \mathrm{MW}$ electrical demand. Hence, the remaining $1.5 \mathrm{MW}$ electrical load demand was purchased from the electricity network to complement with the $4.0 \mathrm{MW}$ electrical load supplied by the CHP. Unfortunately, due to cost of electricity from the market, the total energy cost will increase in this case as seen in Figure 9.

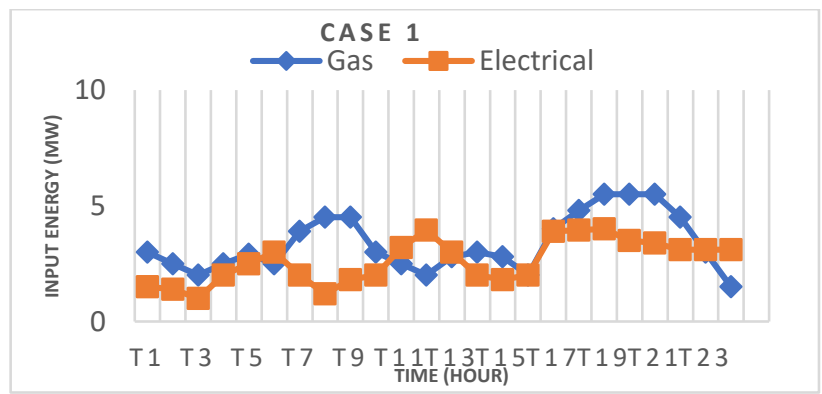

Fig. 5 Total energy input of case 1 in each hour.

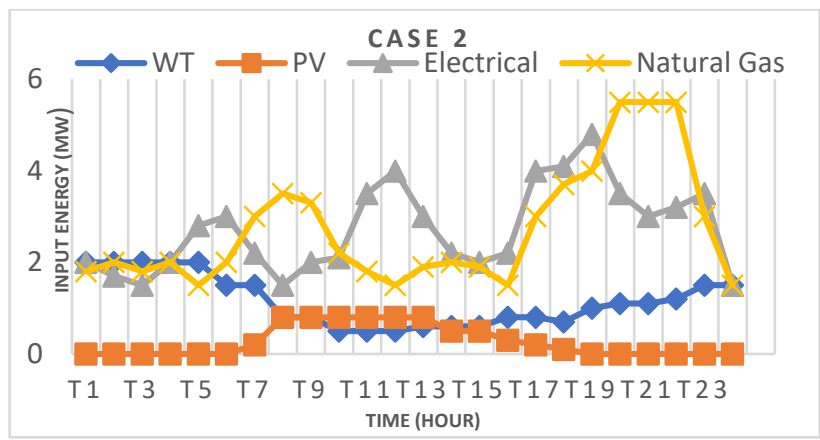

Fig. 6 Input energy of case 2.

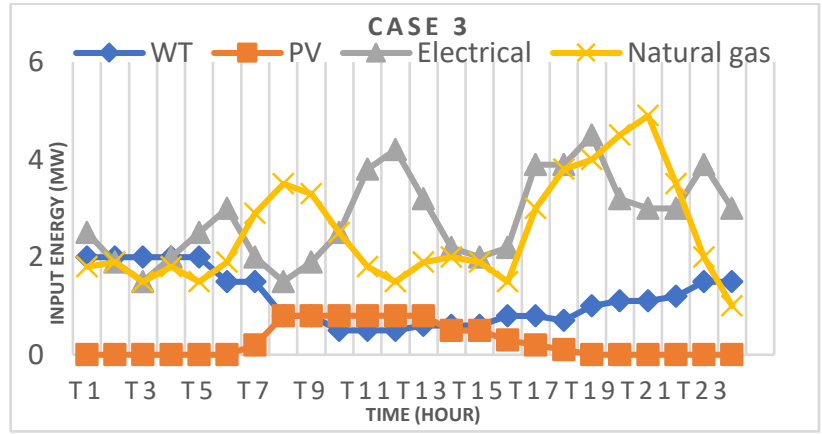


Fig. 7 Input energy of case 3.

When the load is the smallest, the optimal energy flow was chosen in case 2 (i.e., between $1 \mathrm{am}$ to $3 \mathrm{am}$ and $8 \mathrm{am}$ to $11 \mathrm{am}$ ) as seen in figure (3). The CHP was operating at $1.5 \mathrm{MW}$ capacity, and the power capacity of GB is $0.42 \mathrm{MW}$. Additionally, the RESs were introduced to the system in this case to generate extra local electrical power for the EH system. The wind turbine (WT) and the photovoltaic (PV) maximum generation capacity are between $1 \mathrm{am}$ to $5 \mathrm{am}$ and $8 \mathrm{am}$ to $13 \mathrm{pm}$, respectively. Hence, it is assumed that both the WT and PV are generating at their maximum capacity in this case. The WT was operating at $2.0 \mathrm{MW}$, while the PV was operating at $0.8 \mathrm{MW}$. Also, at lower load demand, the electricity price from the market is always cheaper, hence, the EH operator, takes the advantage of lower price to buy more electricity $(1.5 \mathrm{MW})$ from the market. From figure (3) the average load demand between $1 \mathrm{am}$ to $3 \mathrm{am}$ and $8 \mathrm{am}$ to $11 \mathrm{am}$ was between $1.5 \mathrm{MW}$ to $3.5 \mathrm{MW}$. This is because it is assumed that the most consumers are either sleeping or have gone to work. Hence, there is low load demand. In this case, with the utilization of locally clean generated electricity from the WT and PV there is a total of $2.8 \mathrm{MW}$ load from the RESs to the EH. Hence, the total sum of CHP electrical load (0.6MW), the purchased electricity from the network $(1.5 \mathrm{MW})$, and the $2.8 \mathrm{MW}$ load from the RESs was equal to $4.9 \mathrm{MW}$ as against the average load demand of between $1.5 \mathrm{MW}$ to $3.5 \mathrm{MW}$. From the Calculation, the EH operator in this case is left with about $1.4 \mathrm{MW}$ of load that was not consumed. The introduction of RESs have helped the EH operator to purchase less electricity from the market, thereby reducing the EH energy cost. However, the used of RESs in this case have led to the production of high electrical load that is beyond the maximum operating capacity of the EH within the assumed period. This have disrupted the EH operation reliability due to high electricity generation that is beyond the EH demand capacity. To solve this challenge, BESS will be introduced in case 3 to solve the above mention challenges.

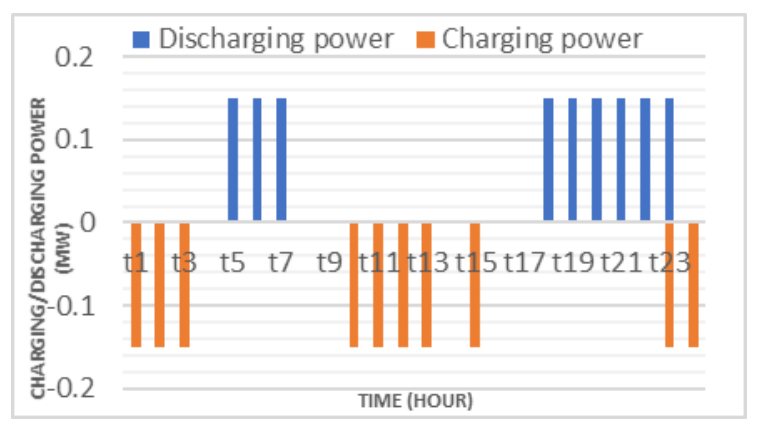

Fig. 8 BESS's charging/discharging power

In case 3, the effect of utilizing BESS locally generated clean renewable energies from WT and PV is analysed on the optimum performance of the EH system. In this case, the BESS was able to store the $1.4 \mathrm{MW}$ excess electrical load that was not utilised in case 2. And discharge the load during the EH higher peak demand (between 5AM, 7 AM, 9 AM and from 5 PM to 10 PM) and the RESs are not at their maximum production capacity due to its variability. This will enable the EH operator to purchase less electrical load from the market as compared to case 1 and 2. With this action, the BESS have not only assisted the EH operator to reduce its energy cost but have also created reliability in the EH system by storing the excess 1.4MW load that have caused disruption to the optimum operation of the EH in case 2. BESS also charges with a maximum power of $0.15 \mathrm{MW}$, which corresponds to the maximum capacity, during the lower electricity pricing hours (from 1AM to 3AM, from 10AM to 1PM, 3PM, and 11PM, 12PM) (8). BESS discharges $0.15 \mathrm{MW}$ back to the $\mathrm{EH}$ system during high electricity pricing hours and high peak hours (at 5 a.m., 7 a.m., 9 a.m., and from 5 p.m. to 10 p.m.). In comparison to cases 1 and 2 , total energy expenses decreased by 21.5 percent. As a result, the EH's total energy expenses in this example are merely $\$ 5572.6$ per 24 hour calculation cycle. Furthermore, by incorporating BESS into the efficient operating of $\mathrm{EH}$ and optimising the energy flow between $\mathrm{CHP}$ and $\mathrm{BESS}$, the total energy purchased by $\mathrm{EH}$ is reduced. The calculation result of overall $\mathrm{EH}$ 
operating costs presented in Fig. 9 reveals that BESS has a significant impact on the EH system's performance. In all three scenarios, the EH optimises the energy flow and kinds. Cases 1 and 2 had operational costs of $\$ 10492.6$ and $\$ 7348.2$, respectively. In case 3, the total cost of energy purchased dropped dramatically to $\$ 5572.6$ or $21.5 \%$ when compared to case 2 .

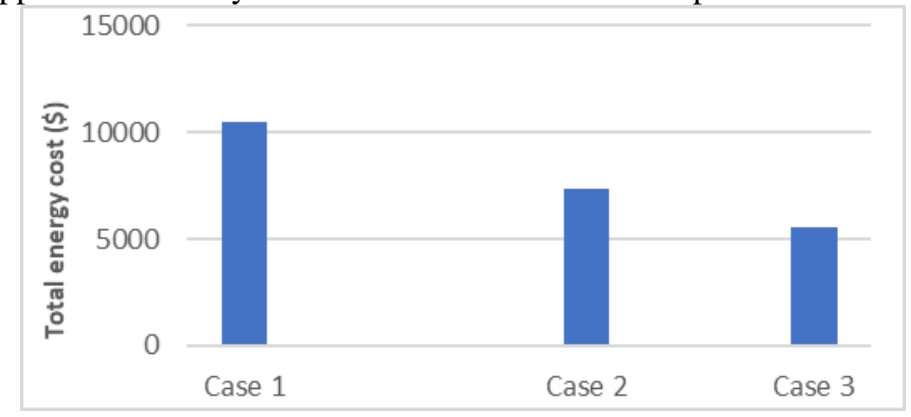

Fig. 9 Total energy cost in the three studied cases

\section{Conclusions}

The following important concerns are addressed in this study:

1. An EH model with numerous loads that is more profitable is proposed. The BESS is taken into account in the model to boost operational flexibility and efficiency. As a result, the EH's economic and technical elements have also improved.

2. Under high shares of RESs and BESS, the EH's ideal operation schedules are computed with the goal of lowering the energy cost purchased from the system. Three case studies with varied architectures have been provided to compare the different EH models that include RESs and BESS. The optimization results demonstrate that by combining an EH with RES generation and a BESS, the overall cost of energy purchased from the market could be reduced by 21.5 percent while completely satisfying demand.. The EH's BESS minimises the amount of electrical energy purchased from the system and lowers the EH's operating costs. As a result, the calculations in this study may be applicable to real-world challenges. Finally, the EH model in this work primarily solves the EH model's optimal operating problem in terms of energy costs. The uncertainties of loads (electricity and heating), electricity prices, and RESs of WT and PV generators, as well as the device investment cost, are not taken into account. In future work, all of the aforementioned topics should be researched further in order to optimise the planning and operation of $\mathrm{EH}$ problems.

\section{References}

[1] S. Shokrzadeh and E. Bibeau, "Sustainable integration of intermittent renewable energy and electrified light-duty transportation through repurposing batteries of plugin electric vehicles," Energy, vol. 106, pp. 701-711, 2016.

[2] H. H. Larsen and L. S. Petersen, "DTU International Energy Report 2015: Energy systems integration for the transition to non-fossil energy systems," 2015.

[3] M. F. Tahir, C. Haoyong, K. Mehmood, N. Ali, and J. A. Bhutto, "Integrated energy system modeling of China for 2020 by incorporating demand response, heat pump and thermal storage," IEEE Access, vol. 7, pp. 40095-40108, 2019.

[4] J. Reynolds, M. W. Ahmad, and Y. Rezgui, "Holistic modelling techniques for the operational optimisation of multi-vector energy systems," Energy and Buildings, vol. 169, pp. 397-416, 2018.

[5] M. Geidl and G. Andersson, "A modeling and optimization approach for multiple energy carrier power flow," in 2005 IEEE Russia Power Tech, 2005: IEEE, pp. 1-7. 
[6] M. Schulze, L. Friedrich, and M. Gautschi, "Modeling and optimization of renewables: applying the energy hub approach," in 2008 IEEE International Conference on Sustainable Energy Technologies, 2008: IEEE, pp. 83-88.

[7] S. M. Ezzati, F. Faghihi, H. M. Shourkaei, S. B. Mozafari, and S. Soleymani, "Optimum operation of multi-energy carriers in the context of an energy hub considering a wind generator based on linear programming," Journal of Renewable and Sustainable Energy, vol. 10, no. 1, p. 014702, 2018.

[8] S. Pazouki, M.-R. Haghifam, and A. Moser, "Uncertainty modeling in optimal operation of energy hub in presence of wind, storage and demand response," International Journal of Electrical Power \& Energy Systems, vol. 61, pp. 335-345, 2014.

[9] S. Pazouki and M. Haghifam, "Market based short term scheduling in energy hub in presence of responsive loads and renewable resources," 2013.

[10] M. Moeini-Aghtaie, A. Abbaspour, M. Fotuhi-Firuzabad, and P. Dehghanian, "Optimized probabilistic PHEVs demand management in the context of energy hubs," IEEE Transactions on Power Delivery, vol. 30, no. 2, pp. 996-1006, 2014.

[11] A. Vian, F. Bignucolo, and M. De Carli, "Modelling and Optimization Approach of Residential Energy Hub: The GHOTEM Project," in 2019 1st International Conference on Energy Transition in the Mediterranean Area (SyNERGY MED), 2019: IEEE, pp. 1-6.

[12] M. Salimi, H. Ghasemi, M. Adelpour, and S. Vaez-ZAdeh, "Optimal planning of energy hubs in interconnected energy systems: a case study for natural gas and electricity," IET Generation, Transmission \& Distribution, vol. 9, no. 8, pp. 695-707, 2015.

[13] A. Y. Abdelaziz, A. M. El-Zonkoly, and A. M. Eladl, "Energy hub optimization using modified firefly algorithm," in 2017 8th International Renewable Energy Congress (IREC), 2017: IEEE, pp. 1-6.

[14] M. Geidl, G. Koeppel, P. Favre-Perrod, B. Klockl, G. Andersson, and K. Frohlich, "Energy hubs for the future," IEEE power and energy magazine, vol. 5, no. 1, pp. 2430, 2006.

[15] L. Ni et al., "Optimal operation of electricity, natural gas and heat systems considering integrated demand responses and diversified storage devices," Journal of Modern Power Systems and Clean Energy, vol. 6, no. 3, pp. 423-437, 2018.

[16] A. Soroudi, Power system optimization modeling in GAMS. Springer, 2017. 